\title{
Dispersive optical phased array circuit for high-resolution pixelated 2D far-field scanning controlled by a single wavelength variable
}

Bogaerts, Wim, Dahlem, Marcus, Dwivedi, Sarvagya, Jansen, Roelof, Rottenberg, Xavier

Wim Bogaerts, Marcus Dahlem, Sarvagya Dwivedi, Roelof Jansen, Xavier Rottenberg, "Dispersive optical phased array circuit for high-resolution pixelated 2D far-field scanning controlled by a single wavelength variable," Proc. SPIE 11284, Smart Photonic and Optoelectronic Integrated Circuits XXII, $112841 Z$ (26 February 2020); doi: 10.1117/12.2544937

SPIE. Event: SPIE OPTO, 2020, San Francisco, California, United States 


\title{
Dispersive optical phased array circuit for high-resolution pixelated 2D far-field scanning controlled by a single wavelength variable
}

\author{
Wim Bogaerts $^{\mathrm{a}}$, Marcus Dahlem ${ }^{\mathrm{b}}$, Sarvagya Dwivedi ${ }^{\mathrm{b}}$, Roelof Jansen ${ }^{\mathrm{b}}$, and Xavier Rottenberg ${ }^{\mathrm{b}}$ \\ ${ }^{a}$ Ghent University - IMEC, Photonics Research Group, Department of Information \\ Technology, Technologiepark-Zwijnaarde 126, 9052 Gent, Belgium \\ bIMEC, Kapeldreef 75, 3001 Leuven, Belgium
}

\begin{abstract}
We propose a 2D scanning optical phased array circuit that reconciliates the requirements of resolution and range in automotive LiDAR. Using only the wavelength of the laser source to scan in both $x$ and $y$ directions, we generate a discrete pixelated pattern in the far field, with both the required resolution and a sufficiently long Rayleigh range. We start from a $2 \mathrm{D}$ dispersive optical phased array consisting of wavelength-dependent grating coupler antennas and long delay lines with sufficient angular resolution, and then combine these into a larger array to achieve the required range specifications.
\end{abstract}

Keywords: Optical Phased Array, Wavelength Steering

\section{INTRODUCTION}

The rapid progress in Advanced Driver-Assistance Systems (ADAS) and autonomous vehicles has spurred the research and development of compact Light Detection and Ranging (LiDAR) solutions, to complement existing in-car sensors and construct a reliable 3D image of the car's surroundings. ${ }^{1-3}$

Approaches are either based on wide-angle illumination of an entire scene ${ }^{4,5}$ or on a scanning approach, ${ }^{6-9}$ where one or more beams are used to scan the reflections in the far field. For automotive applications it is important that, irrespective of the approach, the cost as well as the size, weight, and power (SWAP) of the system is kept within limits. This imposes constraints on the technologies that can be used.

Scanning LiDAR solutions can be based on a variety of technologies, such as mechanical (or MEMS-based) steering, ${ }^{10,11}$ electronic steering or through an optical phased array. ${ }^{9,12,13}$ One approach, which is promising in terms of SWAP as well as cost, is a chip-based, solid-state optical phased array (OPA). Using an on-chip waveguide distribution network and compact on-chip optical antennas, a vertically emitting light pattern is constructed that can be controlled by manipulating the phase differences between the on-chip antennas. ${ }^{14}$ Such a photonic integrated circuit (PIC) can be fabricated using wafer-scale manufacturing technology, in materials such as silicon, silicon nitride, III-V semiconductors or even polymers. ${ }^{15-17}$ In particular, technologies based on silicon or silicon nitride offer a high refractive index contrast which makes the circuits very compact, and these materials can be processed in existing wafer-scale foundries used for CMOS electronics manufacturing. ${ }^{15}$ Several such manufacturing services are available on a commercial or semi-commercial basis. ${ }^{18}$

Several OPA architectures for PIC-based optical beamforming and beamsteering have been proposed and demonstrated. ${ }^{14}$ These are illustrated in Fig. 1. To achieve 1-dimensional beamsteering, a 1D array of off-chip antennas (e.g. grating couplers ${ }^{19,20}$ ) is fed by a distribution network of waveguides, such as a hierarchical tree of $1 \times 2$ splitters (Fig. 1a). ${ }^{12,21}$ While each antenna emits a wide-angle beam along the $x$ direction, the array of antennas will emit a narrow focused beam when the antennas emit with a fixed optical phase delay $\Delta \phi_{x}$ between every two antennas. The emission angle $\theta_{x}$ along the $x$ direction depends on this phase delay $\Delta \phi_{x}$, which can be controlled by an electro-optic phase shifter in front of every antenna. The quality of the composite beam

Further author information: (send correspondence to W.B.)

W.B.: E-mail: wim.bogaerts@UGent.be, Telephone: +32 92643324

Smart Photonic and Optoelectronic Integrated Circuits XXII, edited by Sailing He, Laurent Vivien, Proc. of SPIE Vol. 11284, 112841Z · (c) 2020 SPIE · CCC code: 0277-786X/20/\$21 - doi: 10.1117/12.2544937 
(a)

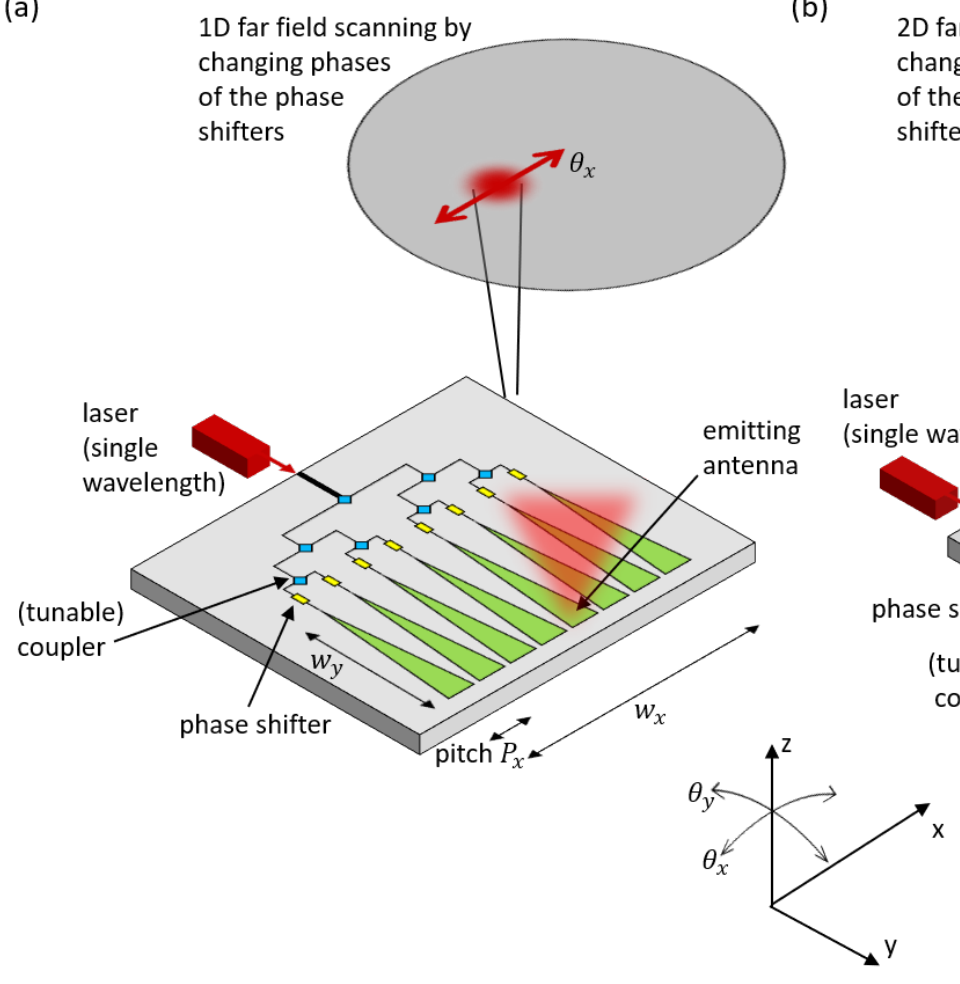

2D far field scanning by changing phases of the phase shifters (c)

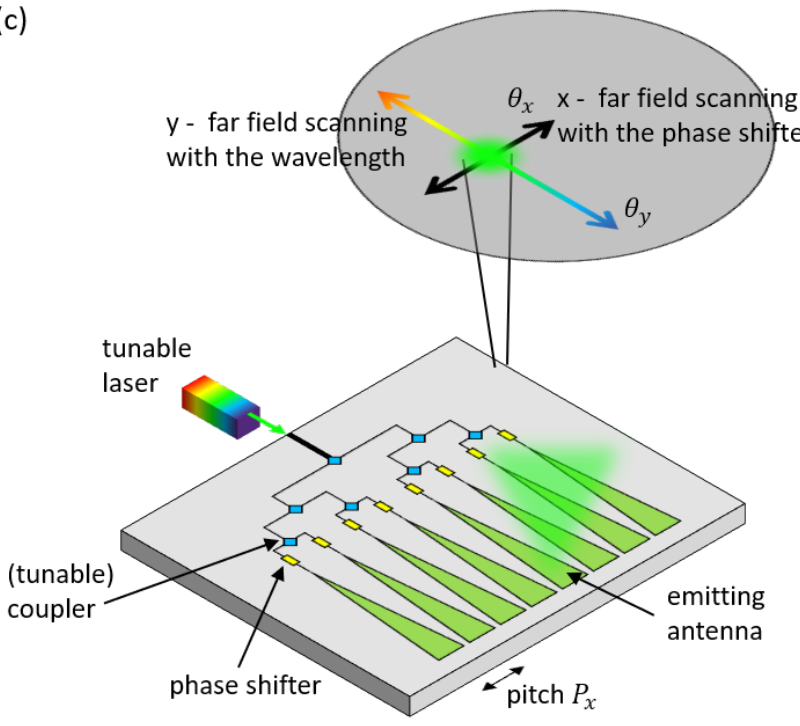

(d)

fast x-scanning with wavelength

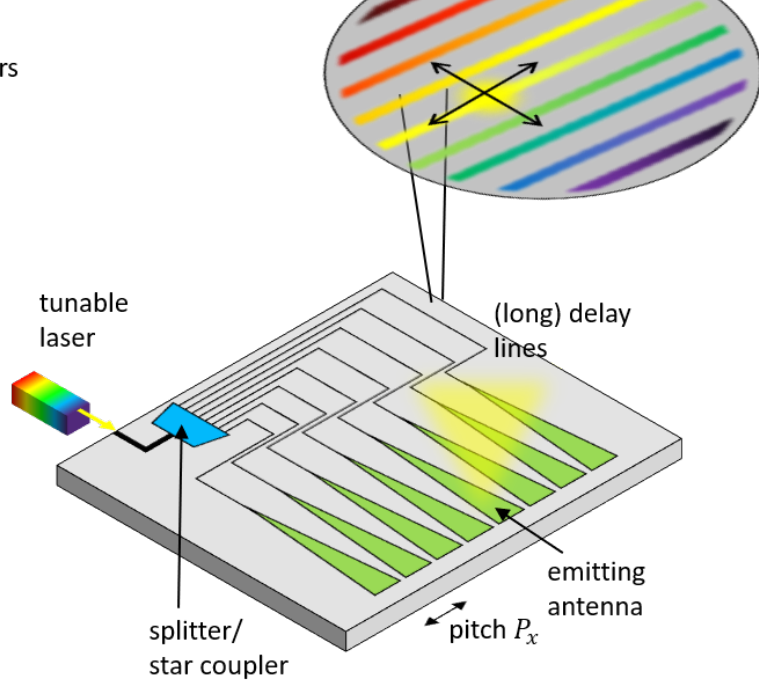

Figure 1. Optical Phased Array Circuits.

depends on the accuracy of the phase control. The available steering angle depends on the period $P_{x}$ between the antennas: the smaller $P_{x}$ with respect to the wavelength of the light, the wider the available steering angle. The divergence of the beam and Rayleigh range $z_{r}$ of the beam are governed by the total size of the array. Therefore, an array which has to emit a narrow beam and at the same time has a wide steering angle, will have a large number of closely spaced antennas. 
The same principle can be applied in two dimensions, as shown in Fig. 1b. Here, the feed network becomes more complicated as it needs to bring light to a 2D array of antennas. By adjusting the relative phases $\Delta \phi_{x}$ and $\Delta \phi_{y}$, the beam can be steered along the $\theta_{x}$ and $\theta_{y}$ directions, respectively. ${ }^{22}$ Because the criteria of divergence and steering angle work along 2 directions, the number of emitting antennas that need phase control can rapidly grow, complicating the control of the overall chip.

An alternative approach for 2D beam steering, illustrated in Fig. 1c, uses the same principle as the 1D beam scanner for scanning along $\theta_{x}$, but it incorporates an antenna which is wavelength dependent, changing the off-chip angle $\theta_{y}$ with wavelength. ${ }^{12,21,23}$ By using a combination of a tunable laser source and the 1-D array of on-chip phase shifters, the emitted beam can be directed in two directions. This simplifies the control significantly compared to the $2 \mathrm{D}$ array.

The use of wavelength for beam steering can be taken one step further, to perform 2D beam scanning using only the wavelength of the input light, as shown in Fig. 1 d. ${ }^{24}$ It uses the same dispersive antennas for scanning along the $\theta_{y}$ direction. However, instead of feeding these antennas with a balanced splitter tree and controlling the phase delay $\Delta \phi_{x}$ through an electro-optic phase shifter, the antennas are connected with an array of long delay lines. These delay lines add an optical delay $\Delta L_{\text {opt }}$ between every two antennas, which translates into a wavelength dependent phase delay $\Delta \phi_{x}$. With long delay lines, this phase delay changes rapidly with wavelength, resulting in a fast beam scan along the $\theta_{x}$ direction. At the same time, the beam is swept slowly along the $\theta_{y}$ direction. This results in a $2 \mathrm{D}$ pattern of scan lines in the far field, controlled by a single variable, i.e. the wavelength of the tunable laser. Therefore we call this architecture a dispersive optical phased array.

While a dispersive OPA seems very attractive, there have been only few demonstrations of this architecture. This is because the basic concept does not scale well for large numbers (hundreds or thousands) of antennas, which would be needed for wide-angle scanning. In this paper, we discuss different implementation strategies of this 2D scanner, and how they scale when we increase the number of antennas. We show that this scaling is unpractical with today's PIC technologies, in terms of optical losses and footprint.

We then propose a modification to the architecture, where we abandon the need for a continuous scanning in the far field, and instead perform a pixelated scan. This makes it possible to scale up the circuit to the LiDAR requirements in ADAS systems.

\section{BEAM SCANNER SPECIFICATIONS}

LiDARs for automotive applications can be categorized into two main specification sets. ${ }^{2}$ Short-range LiDARs are needed to form a 3D image of the vehicle's surroundings in all directions, up to a distance of a few tens of meters. Long-range LiDAR is used for imaging the driving direction and should capture a field-of-view of $50^{\circ}$ horizontal by $20^{\circ}$ vertical. The long-range LiDAR should be able to pick up medium-reflective objects (10\%) up to range of $200 \mathrm{~m}$ and strongly retroreflecting objects (e.g. cat-eyes or microprism-based safety gear with $90 \%$ reflectivity) up to $300 \mathrm{~m}$ away. For a scanning LiDAR, the beam should have a comparable Rayleigh range to avoid excessive diffraction over the projected range.

\subsection{Operational Requirements}

To explore the details of scaling a dispersive OPA, we need to look at the requirements for a beam scanner for ADAS LiDAR. These are illustrated in Fig. 2, and listed in Table 1. If we assume a forward-looking LiDAR, we need to scan a horizontal field of view $\Delta \theta_{x}$ of $50^{\circ}$. Vertically the scan range is lower, with a $\Delta \theta_{y}=20^{\circ}$. To build a sufficiently granular view of the surroundings, and be able to identify pedestrians, cyclists, vehicles and static elements in the scenery, the far field should be scanned with a resolution $\delta \theta_{x}=\delta \theta_{y}=0.1^{\circ}$. This results in an image of $500 \times 200=100000$ pixels. For convenience in further discussions, we will assume that the number of horizontal pixels $n_{x}=512$ instead of 500 .

The LiDAR system should also be able to sense reflections as far away as $z=200 m$, in order to have sufficient response time in fast-paced traffic. This imposes a similar value for the Rayleigh range $z_{r}$ of the beam. We can calculate that this corresponds to a beam divergence of approximately $0.01^{\circ}$, which is $10 \times$ smaller than the requirement for the image resolution in the far field. 


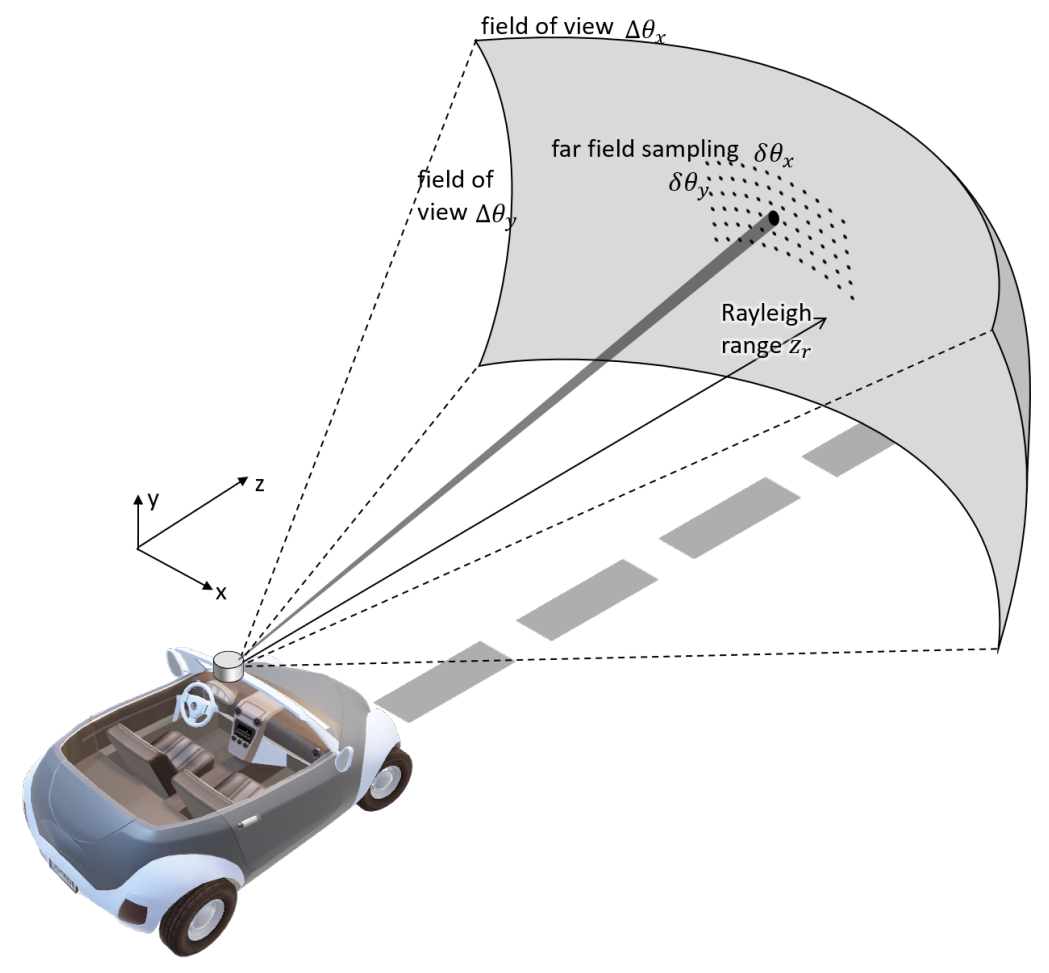

Figure 2. LiDAR in an automotive context. A forward-looking scanning LiDAR should be able to project a beam up to $200 \mathrm{~m}$ over a field-of-view of $50^{\circ} \times 20^{\circ}$, with a sampling resolution of $0.1^{\circ}$.

\subsection{Implementation specifications}

LiDAR systems can be implemented both in visible wavelengths or in the near infrared. For this implementation, we choose to work in the wavelength range between $1525 \mathrm{~nm}$ and $1640 \mathrm{~nm}$. The atmosphere has a low propagation loss (no water absorption) and there are fewer constraints with respect to eye safety, according to regulation IEC/EN 60825. Also, it is possible to build on semiconductor tunable laser technology that has been developed

Table 1. Approximate specifications for the 2D dispersive OPA beam scanner.

\begin{tabular}{|l|r|l|}
\hline Wavelength range $\lambda_{\min }-\lambda_{\max }$ & $1525-1640$ & $n m$ \\
\hline Horizontal field of view $\Delta \theta_{x}$ & 50 & $\circ$ \\
Vertical field of view $\Delta \theta_{y}$ & 20 & $\circ$ \\
\hline Angular sampling resolution $\delta \theta_{x}=\delta \theta_{y}$ & 0.1 & $\circ$ \\
\hline Horizontal pixels $n_{x}$ & 512 & \\
Vertical pixels $n_{y}$ & 200 & \\
\hline Rayleigh Range $z_{r}$ & 200 & $m$ \\
\hline Beam divergence & 0.01 & $\circ$ \\
\hline Emitter width $w_{x}=w_{y}$ & 15 & $m m$ \\
\hline Antenna spacing $P_{x}$ & 1.8 & $\mu m$ \\
\hline Number antennas $N$ for beam divergence & 8192 & \\
Number antennas $P$ for sampling resolution $\delta \theta_{x}$ & 512 & \\
\hline
\end{tabular}


for fiber-optic communication. These wavelengths are compatible with several PIC technologies, and most importantly silicon photonics.

The choice of the wavelength, as well as the field of view, translates into a spacing of the OPA antennas of $P_{x}=1.8 \mu \mathrm{m}$. The requirement of the Rayleigh range $z_{r}=200 \mathrm{~m}$ (and the beam divergence of $0.1^{\circ}$ ) imposes a total size of the emitting area of the OPA of $15 \times 15 \mathrm{~mm}^{2}$. While this can be the basis for a compact LiDAR system, this is a large area for a photonics chip. The combination of small antenna spacing and large emitter area means that the OPA will require $\approx 8000$ antennas. This is a very large number for any of the different implementations illustrated in Fig. 1. Again, for convenience we will dimension the number of antennas $N$ as a power of 2 , so $N=8192$.

The scanning of the beam along the $\theta_{y}$ angle is controlled through the design of the individual antennas. To meet the specifications of the emitting area, each antenna should be $15 \mathrm{~mm}$ long. The antenna should also be sufficiently dispersive that a wavelength sweep of $115 \mathrm{~nm}$ covers the full vertical field of view $\Delta \theta_{y}=20^{\circ}$. In itself, it is a challenging proposition to design grating-based antennas with such specifications, especially in a high-contrast material system such as silicon photonics. ${ }^{25,26}$ In this paper, we will not go deeper into the design of this grating antenna, but we assume that indeed the antenna has the correct dispersion and beam divergence in the $\theta_{y}$ direction.

\subsection{The problem of overdimensioning}

For the discussion in the remainder of the paper, it is important to stress a mismatch in the specifications we have elaborated above. While the requirements on the Rayleigh range and beam divergence require 8000 antennas, this is significantly more than what would be required to collect the $n_{x}=512$ pixels along the $x$-axis. Indeed, if we consider the 500 farfield directions along $\theta_{x}$ as distinct optical spatial modes, we would only require $P=512$ antennas to resolve those modes: every antenna also represents an optical spatial mode, and the OPA essentially acts as a linear mode converter between the 500 antenna modes and the 500 farfield modes.

It is also no coincidence that a surface area corresponding to $P=512$ antennas $(900 \mu \mathrm{m})$ corresponds to a beam divergence of $0.1-0.3^{\circ}$ (depending on the power distribution profile over the 512 antennas), which turns out to be the sampling resolution $\delta \theta_{x}$ in the far field. After all, the OPA is a diffraction-limited system.

This mismatch between the requirement for the Rayleigh range $(N=8192$ antennas $)$ and the sampling resolution is something that we will leverage to scale the dispersive OPA to meet both requirements.

\section{CONTINUOUS 2D DISPERSIVE OPA}

The concept of the dispersive 2D OPA combines a continuous scan along $\theta_{x}$, with discrete scan lines along $\theta_{y}{ }^{24}$ This is again illustrated in Fig. 3. This horizontal scanning is made possible by a set of optical delay lines which introduce an constant optical path delay $\Delta L_{\text {opt }}$ between every two antennas. We will briefly revisit the working principles behind this architecture, and look into three different implementations of these optical paths, and explain how these scale in a different way if we increase the number of antennas.

\subsection{Working Principle}

In the dispersive 2D OPA, the wavelength is used to scan the beam along both $\theta_{x}$ and $\theta_{y}$. The scan along $\theta_{y}$ is engineered through the wavelength dependence of the grating coupler antennas. These are (slightly detuned) second-order waveguide Bragg gratings which diffract light to a near-vertical angle $\theta_{y}$. This angle changes with wavelength, allowing a $\theta_{y}$ sweep by varying the input wavelength. For the further discussion, we assume that the grating is designed such that it provides a $\Delta \theta_{y}=20^{\circ}$ sweep for a wavelength range $\Delta \lambda=115 \mathrm{~nm}$.

The horizontal scan is based on a $1 \mathrm{D}$ optical phased array, where the emitted angle $\theta_{x}$ depends on the phase difference $\Delta \phi_{x}$ between every two neighboring antennas. To sweep over the entire field of view $\Delta \theta_{x}$ we have to scan $\Delta \phi_{x}$ from $-\pi$ to $\pi$. Rather than using active phase shifters, we can introduce this $\Delta \phi_{x}$ by delaying the light with a length $\Delta L_{\text {opt }}$ between every two antennas. The phase delay $\Delta \phi_{x}$ then becomes

$$
\Delta \phi_{x}(\lambda)=2 \pi \cdot \frac{\Delta L_{o p t}(\lambda)}{\lambda}=2 \pi \cdot \frac{\Delta L \cdot n_{e f f}(\lambda)}{\lambda},
$$



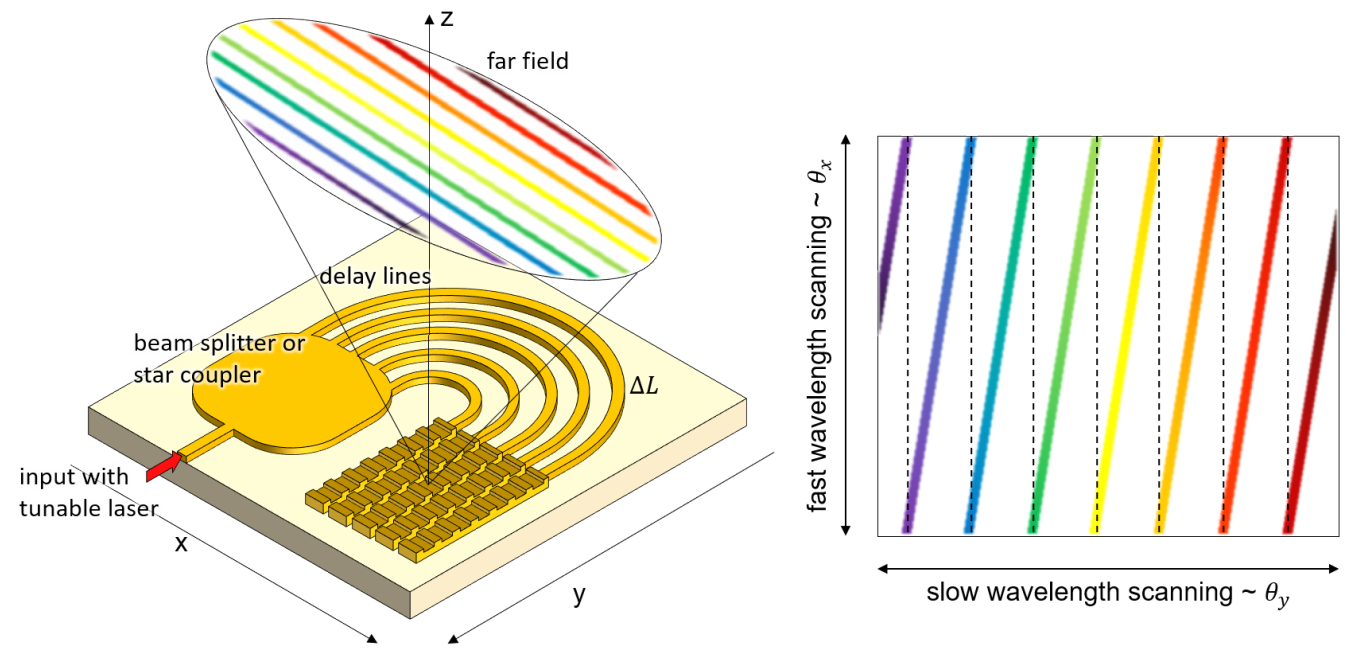

Figure 3. Continuous dispersive 2D optical beam scanner based on an on-chip arrayed waveguide grating, according to Ref. 24.

which is a wavelength-dependent expression, with $n_{e f f}(\lambda)$ as the effective index of the waveguide used in the delay line. The free spectral range (FSR), which is the wavelength range needed to perform a $-\pi \rightarrow \pi$ sweep of $\Delta \phi_{x}$ is

$$
F S R_{x}(\lambda) \approx \frac{\lambda^{2}}{n_{g}(\lambda) \cdot \Delta L}
$$

which will result in a full angular sweep of $\Delta \theta_{x}$ over a single (near-)horizontal scan line. If we now want to implement the specifications discussed in Table 1, we need to fit 200 such scan lines in a wavelength sweep of $\Delta \lambda=115 \mathrm{~nm}$. This means that a single $F S R_{x}$ corresponds to

$$
F S R_{x}=\frac{\Delta \lambda}{n_{y}}=\frac{115 n m}{200}=0.575 n m .
$$

If we implement the delay lines in silicon photonic wire waveguides ${ }^{27}$ with a group index $n_{g} \approx 4.3$, the delay $\Delta L$ between two antennas needs to be slightly larger than $1 \mathrm{~mm}$. While this is not a very long delay line, this is a differential delay, and the longest delay line (of the $N^{\text {th }}$ antenna) has a length of at least $L_{N}=N \cdot \Delta L$, which corresponds to an on-chip delay line of $L_{N}>8 \mathrm{~m}$. Even with low-loss waveguides curled up in spirals, this is excessively long.

\subsection{Implementing the delay lines}

The original concept of the dispersive 2D OPA used an arrayed waveguide grating (AWG) to implement the delay lines. $^{24}$ However, this is only one possible way to implement a differential delay between every two antennas. Three possible implementations have been illustrated in Fig. 4.

The AWG implementation (Fig. 4b) uses a star coupler to split up the light into $N$ waveguides with constant length increments. The total length of waveguide that is needed to implement all delay lines is therefore

$$
L_{t o t, a w g}=\frac{N \cdot(N-1)}{2} \cdot \Delta L,
$$

which scales with the square of the number of antennas $N$. This is a waste of floor space: while all the light has to travel through the first delay length, it is doing this through $N$ different waveguides. 
(a)
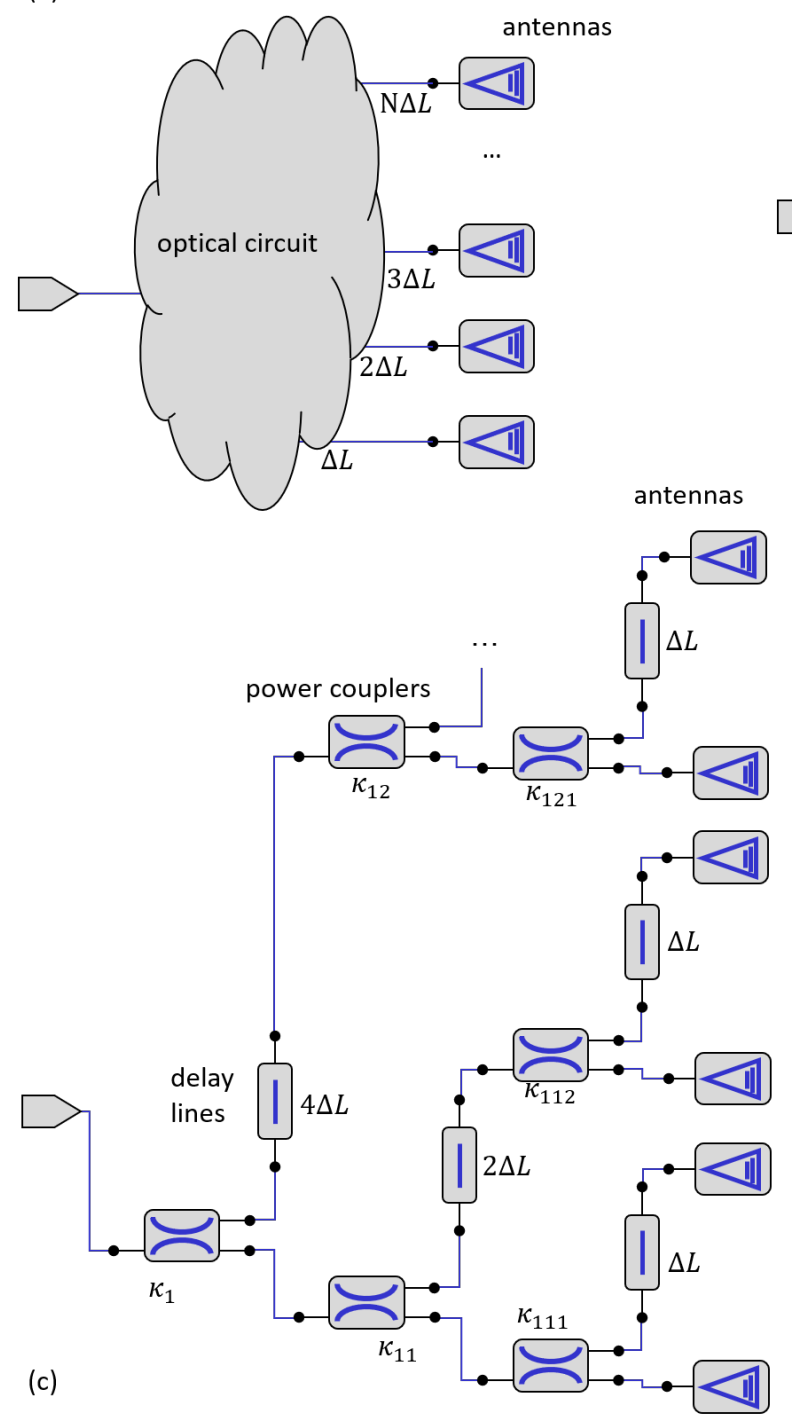

(b)
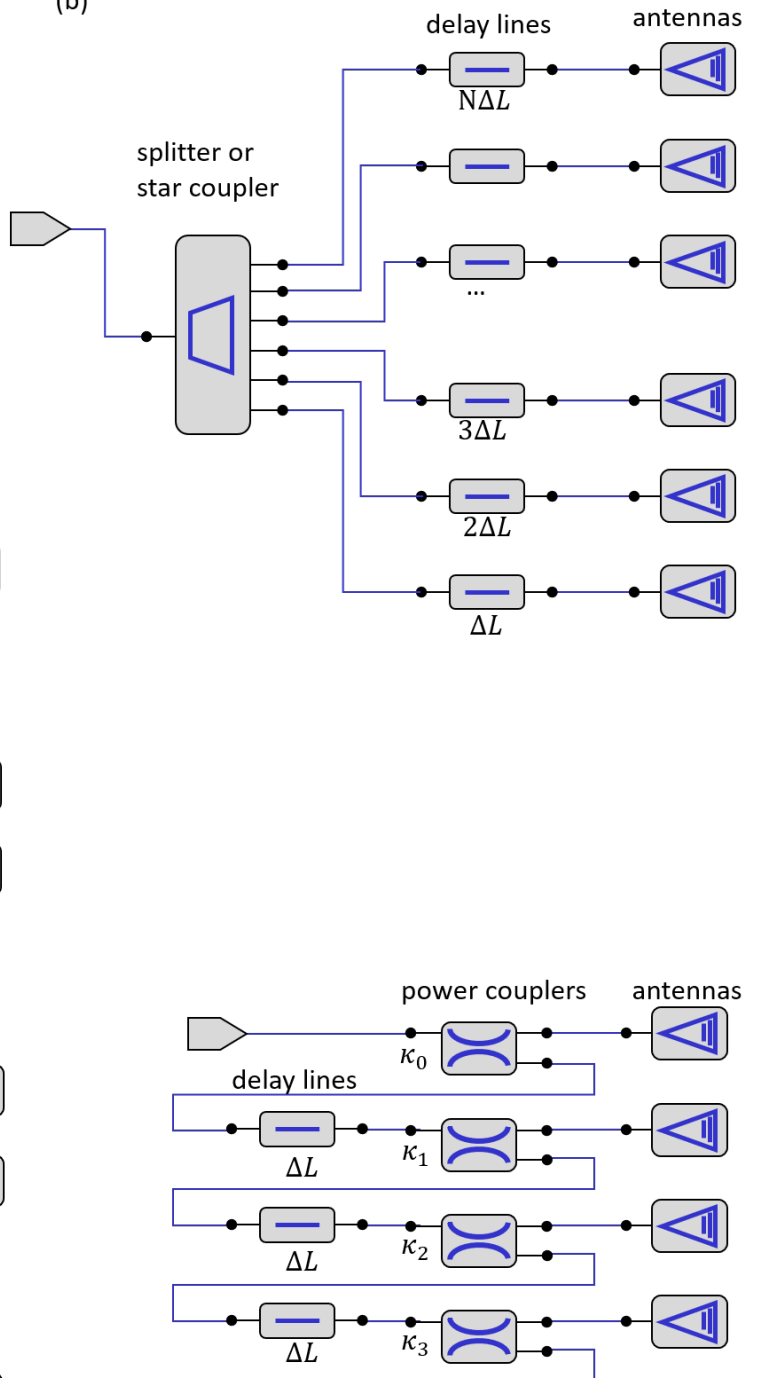

(d)

$\cdots$

Figure 4. Different implementations of the delay lines for the 2D dispersive OPA. (a) The requirement specifies that the differential delay between two antennas is equal to $\Delta L$. (b) The original implementation ${ }^{24}$ uses a star coupler and an arrayed waveguide grating (AWG) with parallel waveguides with incremental length increase. (c) A tree implementation using properly balanced splitters and a logarithmic staggering of delays. (d) A snake implementation where a chosen fraction of light is coupled from a single bus waveguide, with a fixed delay $\Delta L$ between every two couplers.

The snake implementation, shown in Fig. 4d, fixes this problem. Here, all light propagates through the same bus waveguide, and light is tapped off at each antenna, with a delay $\Delta L$ between every two antennas. This tap can be done with a simple (tunable) directional coupler, or the antenna itself can be integrated in the waveguide and tap off a small amount of light. ${ }^{28}$ Now, the total length of waveguide that needs to be laid out on the chip is only

$$
L_{\text {tot, snake }}=N \cdot \Delta L .
$$

While the snake layout makes much better use of the waveguides, it is not straightforward to engineer all the taps to couple the exact amount of power from the waveguide. Also, all the optical power is concentrated 
(a)

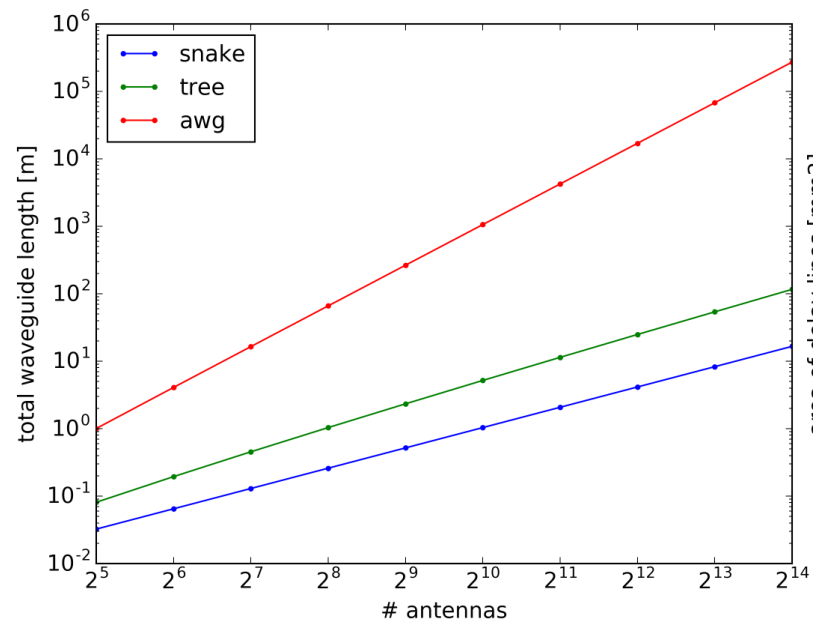

(b)

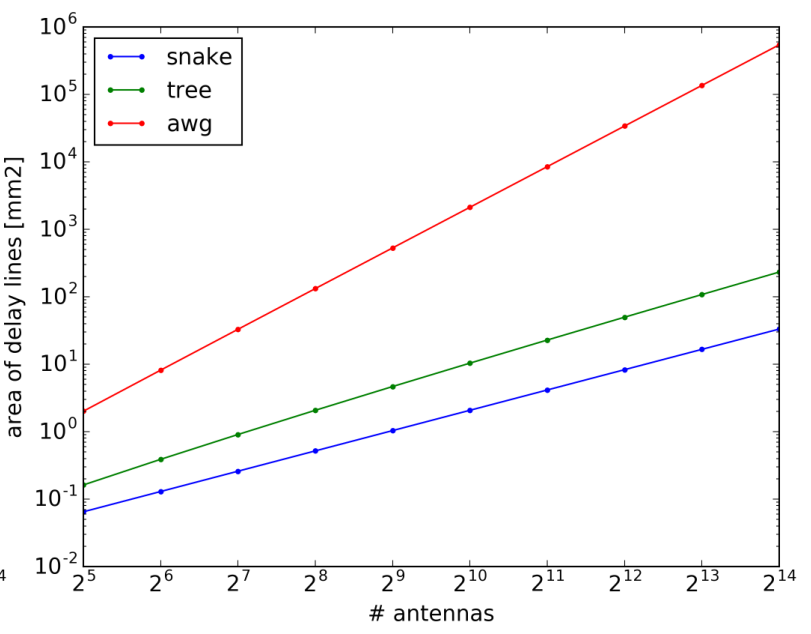

Figure 5. Scaling of the different dispersive OPA layouts from Fig. 1 as function of the number of antennas $N$. (a) The total delay length for all antennas, and (b) the area of the delay lines, assuming $2 \mu m$ waveguide spacing.

in a single waveguide, which could give rise to unwanted nonlinear effects if high power is needed for LiDAR applications.

The tree layout (Fig. 4c) situates itself between the AWG and the snake layout. It consists of a splitter tree, where at each level a delay line is added with doubling lengths $(\Delta L, 2 \Delta L, 4 \Delta L, \ldots)$. The result is the desired incremental delay between adjacent antennas. In the tree layout, delays are largely shared, but not to the extent as in the snake. The total waveguide length for the tree scales as

$$
L_{\text {tot }, \text { tree }}=\frac{N \cdot \log _{2} N}{2} \cdot \Delta L .
$$

The easiest implementation of the tree is if the number of antennas is a power of 2 . That is why, for the comparison, we have chosen $N=8192=2^{13}$ and $P=512=2^{9}$.

\subsection{Scaling of the Dispersive OPA}

The different length scaling of the AWG, snake and tree implementations has its impact on the optical losses of the OPA and the on-chip footprint needed to lay out the delay lines. The total delay length as function of $N$ is plotted in Fig. 5, as well as the minimal required area assuming a tight waveguide spacing $s=2 \mu m$. The quadratic scaling of the AWG layout consumes impractical amounts of floor space for larger arrays, while the tree and the snake stay well within manufacturable chip areas, even for thousands of antennas.

While it seems that these two architectures can scale to larger arrays, the cumulative waveguide losses of the long delay lines, with a $\Delta L=1.016 \mathrm{~mm}$ (corresponding the $F S R_{x}$ calculated above), become excessively high. The scaling of these losses are plotted in Fig. 6. We see that, surprisingly, the losses for the snake, with its shorter cumulative delay lines, are higher than for the tree. This can be explained by the fact that in the snake layout the light, on average, has to pass through $N / 2$ power couplers that tap off the light, while in the tree layout the light only passes through $\log _{2} N$ couplers. Even with a low coupler loss of only $-0.02 \mathrm{~dB}$, the additional losses that come from the couplers will dominate in the snake layout.

We also see from Fig. 6 that for high values of $N$ the losses become unmanageable, unless waveguide losses can be reduced to $5 \mathrm{~dB} / \mathrm{m}$ or less. In submicron silicon waveguides (which we assumed here to allow for a tight waveguide spacing of $2 \mu \mathrm{m}$ ), practical propagation losses are still 1-2 orders of magnitude larger. Given practical technology constraints, the number of antennas is therefore limited to 512 . 
(a)

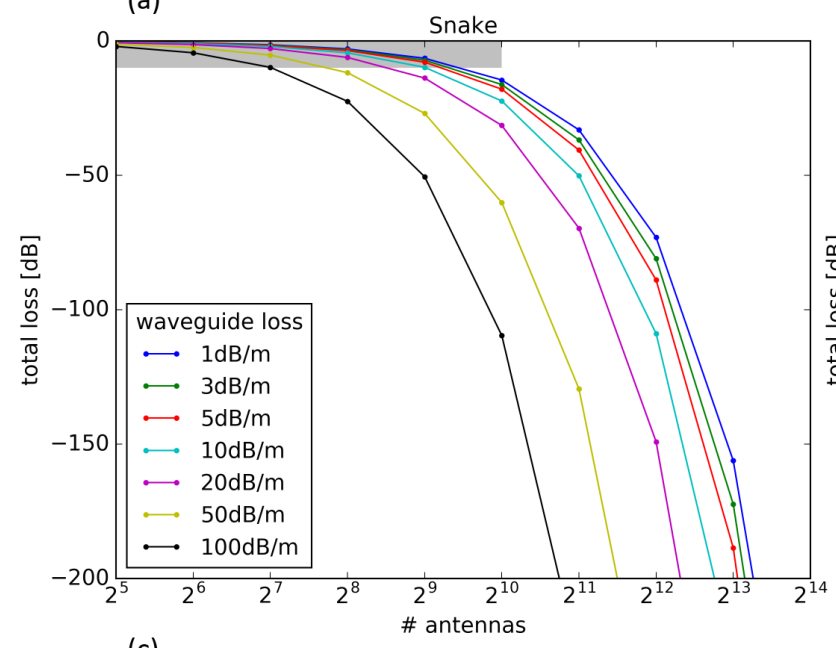

(c)

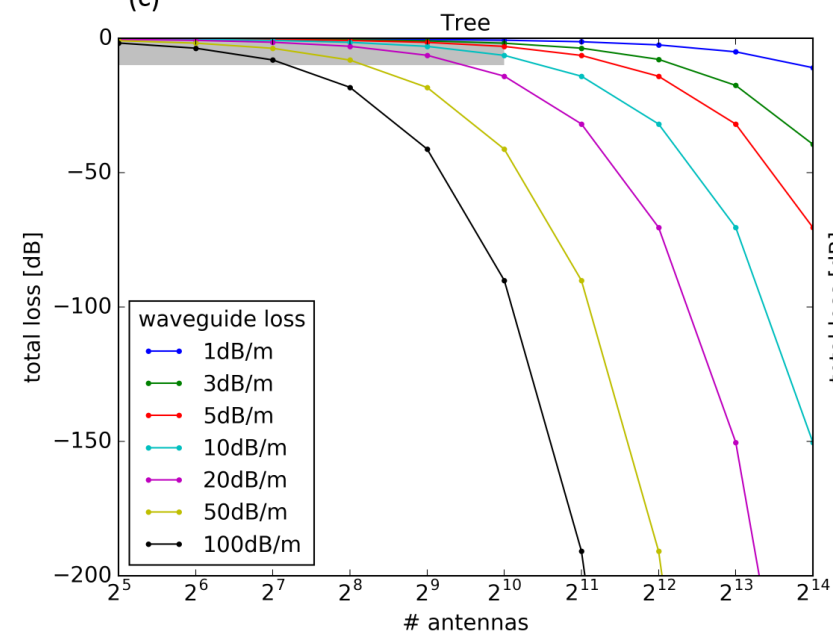

(b)

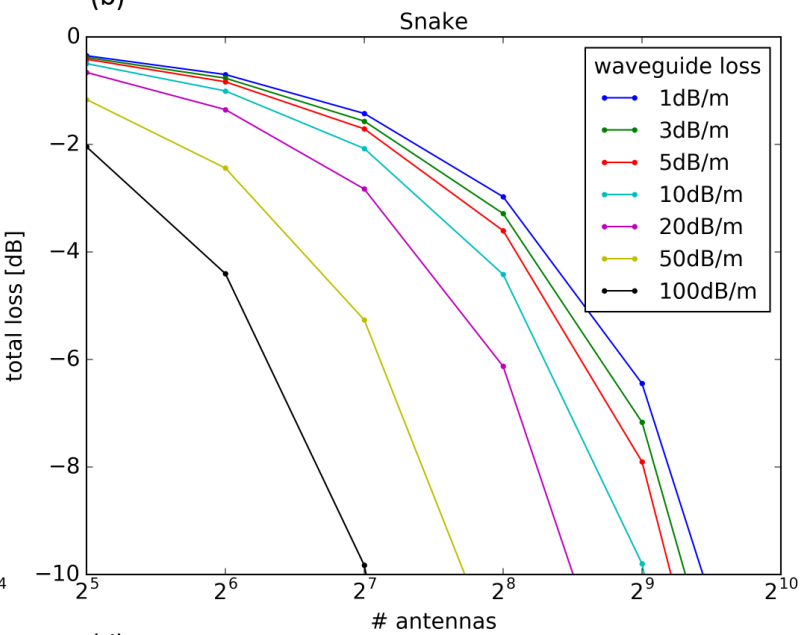

(d)

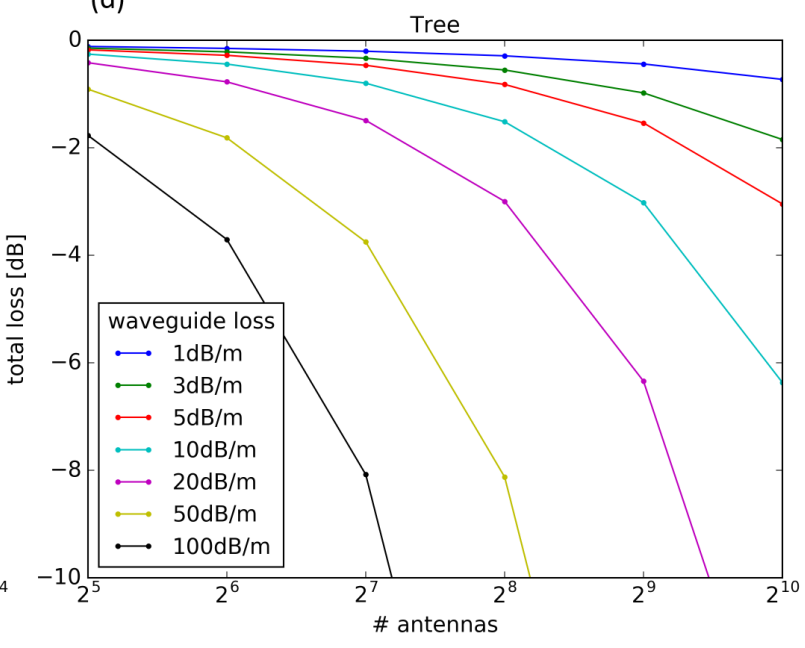

Figure 6. Scaling of the cumulative optical losses in the snake and tree-type OPAs in Fig. 1. (a) Loss in the snake OPA as function of the number of antennas $N$ for different waveguide losses. (b) Zoom of the shaded region in (a). (c-d) Same losses for the tree-type OPA. Each tap or coupler in the circuit also introduces $-0.02 \mathrm{~dB}$ loss.

\section{DISCRETIZED 2D DISPERSIVE OPA}

To respect the scaling boundaries of the $2 \mathrm{D}$ dispersive OPA, but at the same time meet the requirements layed out in section 2, we revisit the discrepancy between the beam divergence of $0.01^{\circ}$ which was dictated by the Rayleigh range $z_{r}=200 \mathrm{~m}$, and the sampling resolution $\delta \theta_{x}=0.1^{\circ}$. While the former requires $N=8192$ antennas, the latter, more relaxed criterion only requires $P=512$ antennas, which is achievable with the snake and tree layout.

\subsection{Operational Principles}

How to scale up the dispersive OPA to $N=8192$ antennas to make sure the beam is sufficiently narrow? The solution we propose here is illustrated in Fig. 7. Instead of building a single dispersive 2D OPA from $N=8192$ antennas with increasingly long delay lines, we construct a dispersive OPA block with only $P=512$ antennas, and stack $M=16$ of those blocks together into a larger antenna array of $N=M \times P=8192$ antennas. The smaller blocks can be implemented as an AWG, tree or snake. ${ }^{29}$ Essentially, this system now becomes a phased array of $M=16$ large antennas, which each emit a beam with $0.1-0.3^{\circ}$ divergence (the divergence depends 


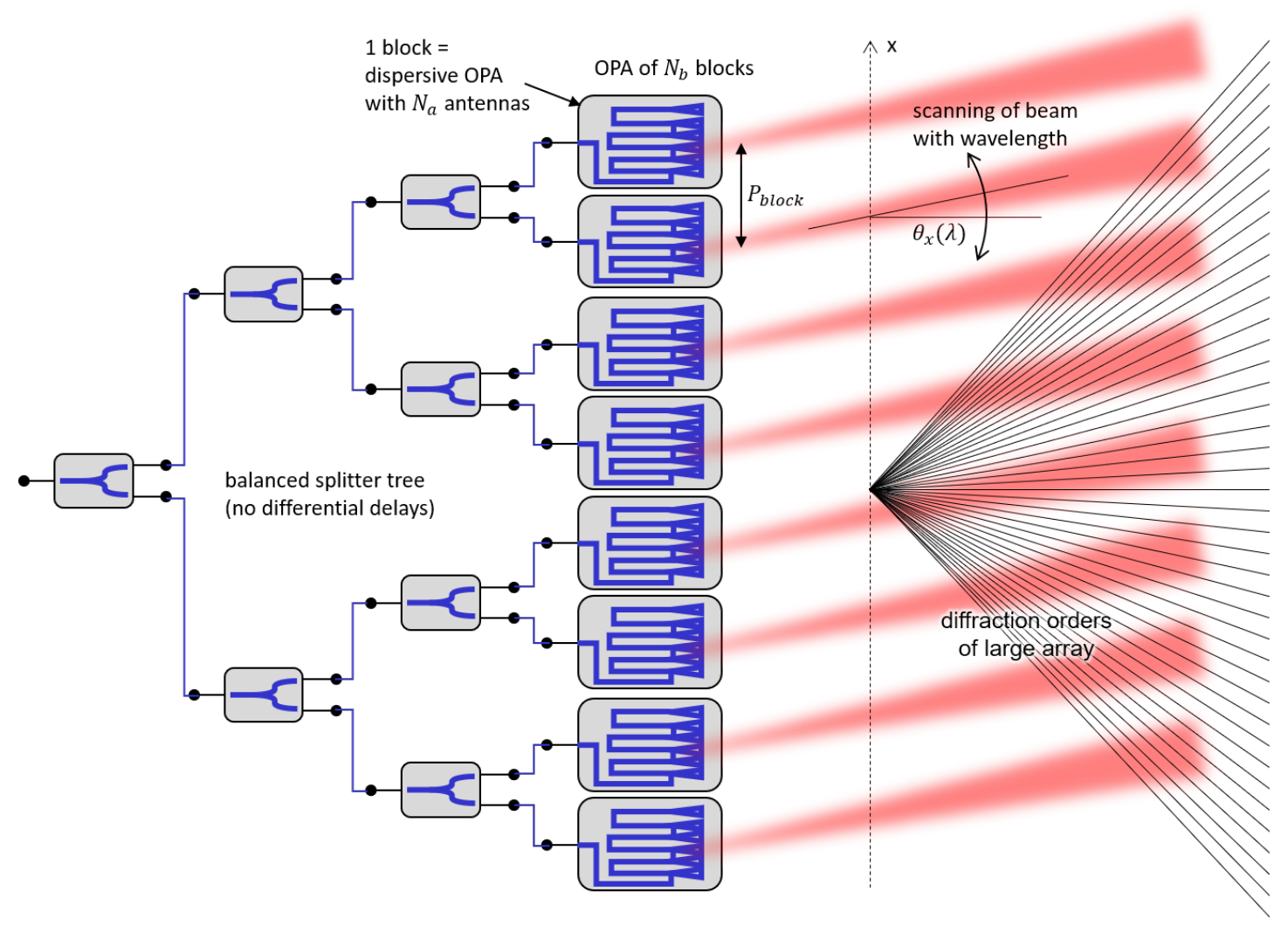

Figure 7. Principle of the discretized 2D dispersive OPA. $M$ blocks of $P$ antennas are stacked together into a large array of $N=M \times P$ antennas. The emitted beam of the $M$ blocks is scanning as function of wavelength, but the $M$ beams only interfere constructively on one of the $P$ diffraction orders of the larger OPA.

on the power distribution within each large antenna block), which sweeps rapidly along $\theta_{x}$ when the wavelength changes within a single $F S R_{x}$.

The compound OPA, which has a very large spacing between its $M$ block antennas, obviously has a large number $P$ of diffraction orders within the field of view $\Delta \theta_{x}$. The emitted beams of the $M$ blocks will only be in phase when they align with one of these $P$ diffraction orders. Outside of these diffraction orders, all $M$ beams still emit in the same direction, but they are not in phase and therefore do not constructively interfere into a narrow focused beam. Because of the way we constructed the compound phased array, there are approximately 512 diffraction orders in the $50^{\circ}$ field of view (the exact number depends on the wavelength), spaced $\approx 0.1^{\circ}$ apart.

This can also be understood from Fig. 8, which separates the element factor and the array factor of the compound OPA. The element factor is wavelength dependent, as each block is essentially a $2 \mathrm{D}$ dispersive OPA which scans the far field along $\theta_{x}$ (fast) and $\theta_{y}$ (slow). The array factor consists of $P$ diffraction orders, which move slowly apart for longer wavelengths. The resulting far-field pattern, as function of wavelength, is the product of these two. It consists of a $2 \mathrm{D}$ arrangement of pixels where the beam will be focused.

\subsection{Calculations}

We calculated the response of both the continuous and the discretized dispersive OPA by simulating the far field of a single antenna and then applying the array factor for the $N=8192$ antennas with the phases incurred in the delay lines.

For these calculations, we did not assume any propagation losses in the waveguides (these have been discussed above), and for simplicity of the comparison we assumed a uniform power distribution over all antennas. The 

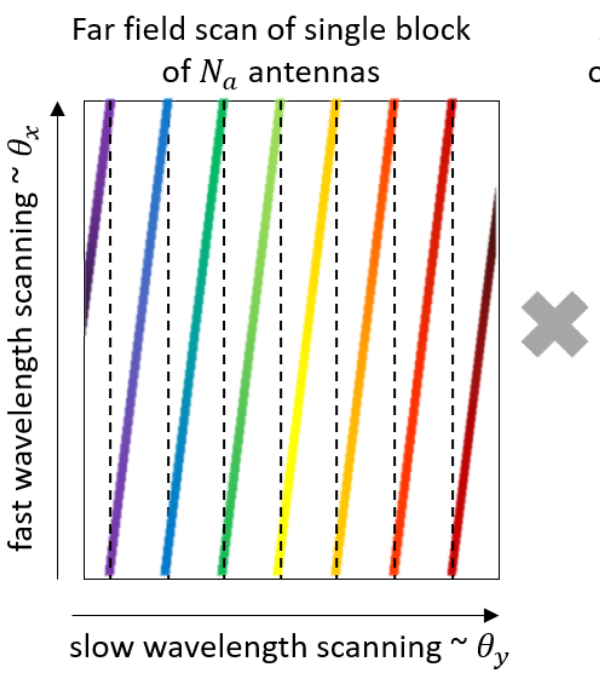

Array factor: diffraction order of $N_{b}$ blocks with period $P_{\text {block }}$

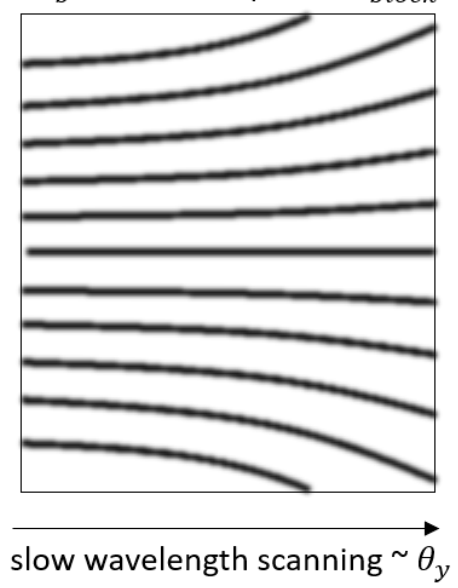

Total emission pattern with wavelength scan

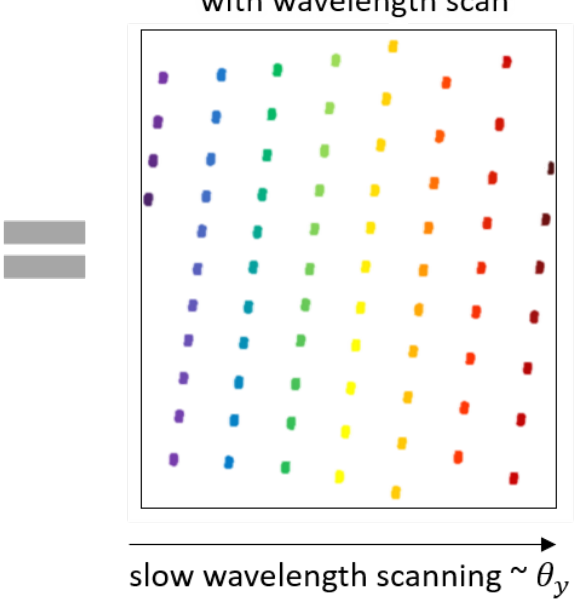

6

Figure 8. Farfield pattern of the discretized 2D OPA, derived from the element factor of each of the $M$ blocks, and the array factor of the periodic array. The resulting pattern in the far field is a $2 \mathrm{D}$ array of pixels where all $N$ emitters are in phase.

main parameters used in the simulations are listed in Tables 1 and 2.

Fig. 9 shows a detail of the far field along $\theta_{x}$ for 5 wavelengths near the central wavelength of $\lambda=1582 \mathrm{~nm}$. For the continuous dispersive OPA (right plots), we see, as expected, that the farfield pattern just moves smoothly along $\theta_{x}$ as we change the wavelength. For the discretized dispersive OPA (left plots), this is not the case. Due to the compound phased array effect, we see pronounced peaks at discrete intervals of $0.1^{\circ}$. As the wavelength changes, the power distribution over those peaks changes. Only when all antenna elements are in phase, the pattern becomes identical to that of the continuous OPA and the other peaks are fully suppressed.

Because we used a uniform power distribution over all antennas, the farfield pattern will be a $\sin \left(\theta_{x}\right) / \theta_{x}$ (sinc) pattern, which we can see as the smaller sidelobes. For the discretized OPA, these sidelobes remain in place, and their number between every two main peaks is $M-2$. When all antennas are in phase, the other large peaks break down to two sidelobe peaks.

We see that the separation of the main peaks corresponds to our calculated spacing $\delta \theta_{x} \approx 0.1^{\circ}$. Because this spacing is linked to the ratio between the wavelength and the period $P_{x}$ of the array, the spacing will slowly increase for longer wavelengths.

Table 2. Example parameters of a discretized 2D dispersive OPA

\begin{tabular}{|l|r|l|}
\hline Number of antennas $P$ in a block & 512 & \\
Number of blocks $M$ & 16 & \\
Total number of antennas $N$ & 8192 & \\
\hline Antenna spacing $P_{x}$ & 1.8 & $\mu \mathrm{m}$ \\
\hline Free spectral range $F S R_{x}$ & 0.575 & $\mathrm{~nm}$ \\
Waveguide group index $n_{g}$ & 4.3 & \\
Delay line $\Delta L$ & 1.016 & $\mathrm{~mm}$ \\
\hline
\end{tabular}



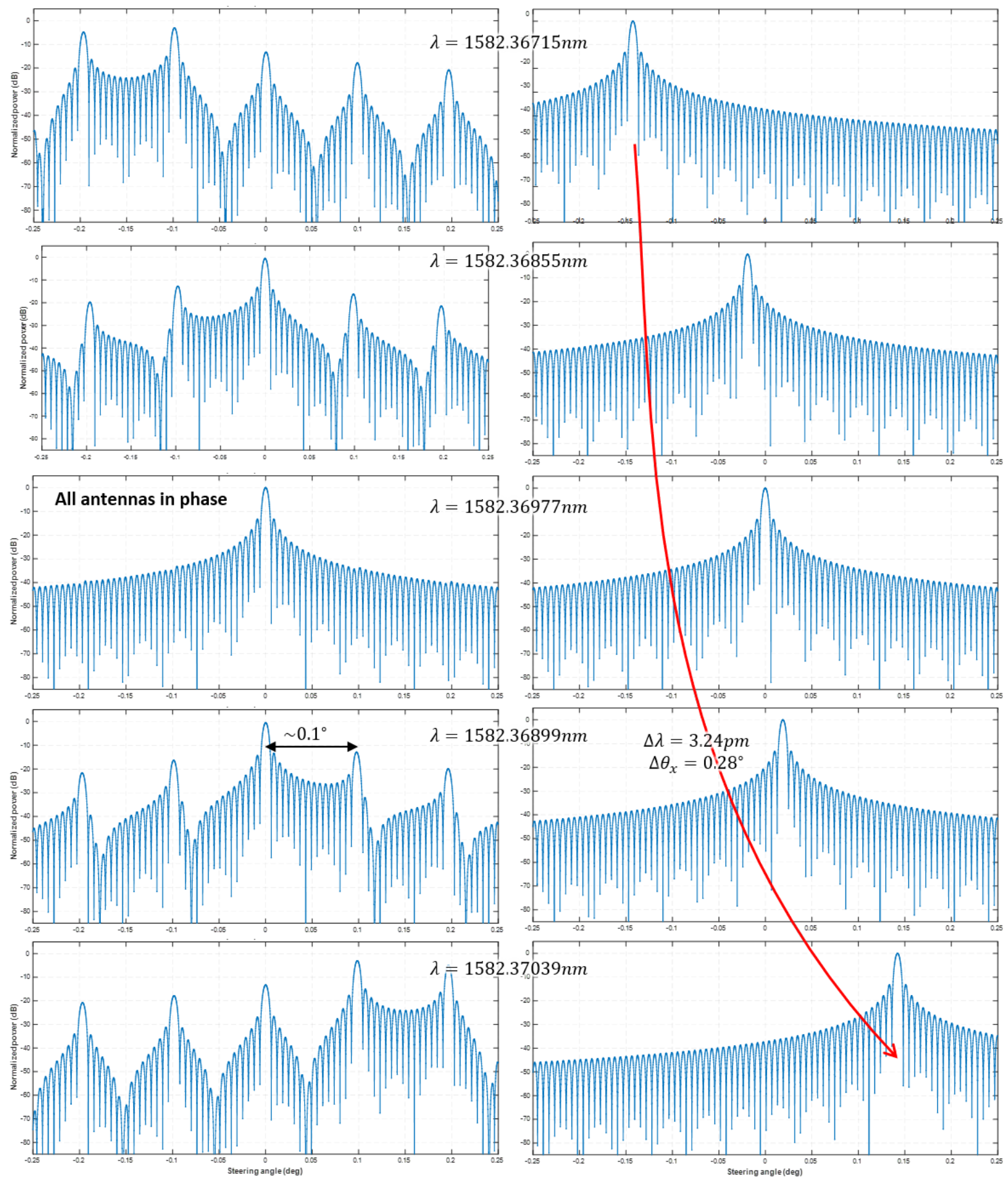

Figure 9. Farfield patterns of both the continuous and the discretized dispersive OPA with the design details listed in Tables 1 and 2. The plots show a detail of the far field along $\theta_{x}$ direction for 5 different wavelengths around the constructive interference condition occuring at $\lambda=1582.36977 \mathrm{~nm}$. The discretized OPA (left) has peaks in fixed locations corresponding to the diffraction orders of the larger array of blocks. As the wavelength changes, power is transferred from one peak to the next. The continuous OPA (right) sees the peak moving continously with wavelength. 


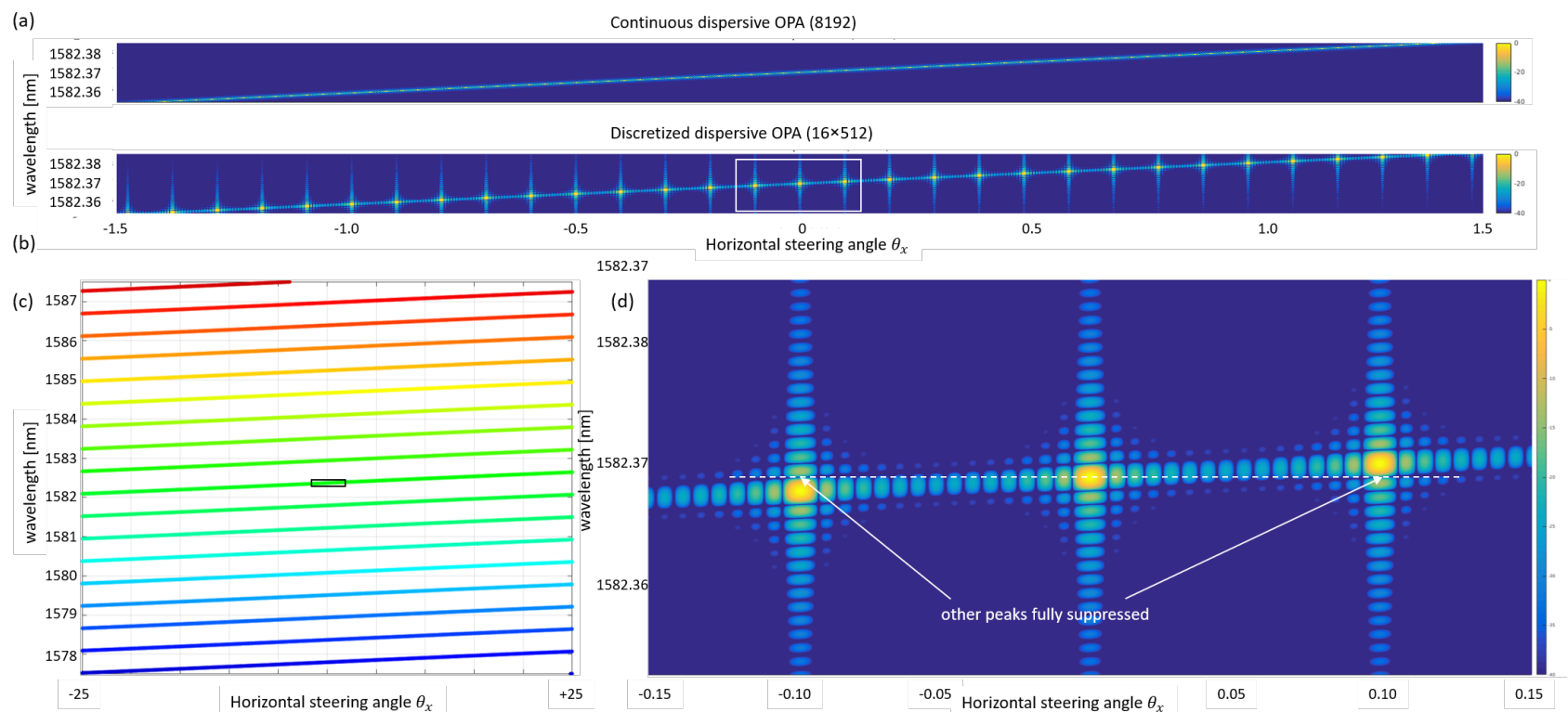

Figure 10. Farfield pattern along $\theta_{x}$ as function of wavelength. (a) Farfield for the continuous dispersive OPA, moving smoothly along $\theta_{x}$ with increasing wavelength (the small dots in the plot are rendering artifacts). (b) The same scan for the discretized dispersive OPA, which clearly shows the discrete peaks. (c) Path along $\theta_{x}$ as function of wavelength for both the discretized and continuous OPA, for a wavelength range of $\pm 5 \mathrm{~nm}$ around the center wavelength. (d) Close-up of the the white rectangle in (b).

Fig. 10c shows a wavelength scan of $\pm 5 \mathrm{~nm}$ around the central wavelength. When we zoom in on a detail of the far-field profile, we see again that the continuous OPA (Fig. 10a) smoothly scans along $\theta_{x}$ with wavelength, while for the discretized OPA (Fig. 10b) we clearly see the discrete peaks with their sinc-like diffraction pattern. In the detail of Fig. 10d we can see that, at the condition where all antennas are in phase, the other peaks are suppressed.

\section{CONCLUSION}

We presented a concept to scale up an optical phased array based on dispersive delay lines. To overcome the overall losses in the waveguides, we separated the requirements for beam divergence (Rayleigh range) and for sampling resolution. By limiting the size of the dispersive OPA to that required by the sampling resolution, we could drastically reduce the amount of waveguide delay lines. And to meet the range requirement, we then combined multiple dispersive OPAs into a larger antenna supplied by a balanced splitter tree without dispersive delays. For the dispersive OPA blocks, we quantitatively compared two architectures based on a tree and snake topology.

This approach results in a farfield pattern with discretely illuminated pixels. When we scan the wavelength, the peak optical power will be transferred from pixel to pixel. We illustrated this with an example of 8192 antennas, sufficient to scan a $\pm 25^{\circ}$ horizontal and $\pm 10^{\circ}$ vertical field of view with a resolution of $0.1^{\circ}$ when scanning the wavelength from 1525 to $1640 \mathrm{~nm}$.

\section{ACKNOWLEDGMENTS}

The authors would like to acknowledge Scantinel Photonics (www.scantinel.com) for their support and funding.

\section{REFERENCES}

[1] Behroozpour, B., Sandborn, P. A., Wu, M. C., and Boser, B. E., "Lidar System Architectures and Circuits," IEEE Communications Magazine 55(10), 135-142 (2017). 
[2] Hecht, J., "Lidar for self-driving cars," Optics and Photonics News 29(1), 26-33 (2018).

[3] Pulikkaseril, C. and Lam, S., "Laser eyes for driverless cars: the road to automotive LIDAR," in [Optical Fiber Communication Conference (OFC) 2019], Tu3D.2, Optical Society of America (2019).

[4] Stettner, R., "Compact 3D flash lidar video cameras and applications," in [Laser Radar Technology and Applications XV], Turner, M. D. and Kamerman, G. W., eds., 7684, 39-46, International Society for Optics and Photonics, SPIE (2010).

[5] Hutchings, S. W., Johnston, N., Gyongy, I., Al Abbas, T., Dutton, N. A. W., Tyler, M., Chan, S., Leach, J., and Henderson, R. K., "A Reconfigurable 3-D-Stacked SPAD Imager With In-Pixel Histogramming for Flash LIDAR or High-Speed Time-of-Flight Imaging," IEEE Journal of Solid-State Circuits 54, 2947-2956 (11 2019).

[6] Weimer, C., Ramond, T., Adkins, C. M., Applegate, J. T., Delker, T. P., and Ruppert, L. S., "Electronically steered flash LIDAR," (5 2014).

[7] Craig, G., "Rigorous 3D error analysis of kinematic scanning LIDAR systems," Journal of Applied Geodesy 1(3), 147-157 (2008).

[8] Wallace, L., Lucieer, A., Watson, C., and Turner, D., "Development of a UAV-LiDAR System with Application to Forest Inventory," Remote Sensing 4, 1519-1543 (5 2012).

[9] Xie, W., Komljenovic, T., Huang, J., Tran, M., Davenport, M., Torres, A., Pintus, P., and Bowers, J., "Heterogeneous silicon photonics sensing for autonomous cars," Opt. Express 27, 3642-3663 (2 2019).

[10] Hu, Q., Pedersen, C., and Rodrigo, P. J., "Eye-safe diode laser Doppler lidar with a MEMS beam-scanner," Optics Express 24(3), 1934 (2016).

[11] Smith, B., Hellman, B., Gin, A., Espinoza, A., and Takashima, Y., "Single chip LIDAR with discrete beam steering by digital micromirror device," Optics InfoBase Conference Papers Part F66-F(13), 14732-14745 (2017).

[12] Van Acoleyen, K., Bogaerts, W., Jágerská, J., Le Thomas, N., Houdré, R., and Baets, R., "Off-chip beam steering with a one-dimensional optical phased array on silicon-on-insulator," Optics Letters 34(9), 1477 (2009).

[13] Watts, M. R., "Towards an Integrated Photonic LIDAR Chip," in [Imaging and Applied Optics 2015], AIW4C.1, Optical Society of America (2015).

[14] Heck, M. J., "Highly integrated optical phased arrays: Photonic integrated circuits for optical beam shaping and beam steering," Nanophotonics 6(1), 93-107 (2017).

[15] Chen, X., Milosevic, M. M., Stankovic, S., Reynolds, S., Bucio, T. D., Li, K., Thomson, D. J., Gardes, F., and Reed, G. T., "The Emergence of Silicon Photonics as a Flexible Technology Platform," Proceedings of the IEEE 106(12), 2101-2116 (2018).

[16] Smit, M., Williams, K., and van der Tol, J., "Past , present , and future of InP-based photonic integration," APL Photonics 050901(May) (2019).

[17] De Felipe, D., Kleinert, M., Zawadzki, C., Polatynski, A., Irmscher, G., Brinker, W., Moehrle, M., Bach, H. G., Keil, N., and Schell, M., "Recent Developments in Polymer-Based Photonic Components for Disruptive Capacity Upgrade in Data Centers," Journal of Lightwave Technology 35(4), 683-689 (2017).

[18] Rahim, A., Spuesens, T., Baets, R., and Bogaerts, W., "Open-Access Silicon Photonics: Current Status and Emerging Initiatives," Proceedings of the IEEE 106(12), 2313-2330 (2018).

[19] Taillaert, D., Bogaerts, W., Bienstman, P., Krauss, T. F., Van Daele, P., Moerman, I., Verstuyft, S., De Mesel, K., and Baets, R., "An out-of-plane grating coupler for efficient butt-coupling between compact planar waveguides and single-mode fibers," IEEE Journal of Quantum Electronics 38(7), 949-955 (2002).

[20] Roelkens, G., Vermeulen, D., Van Thourhout, D., and Baets, R., "High efficiency diffractive grating couplers for interfacing a single mode optical fiber with a nanophotonic silicon-on-insulator waveguide circuit," Appl. Phys. Lett. 92(13), 131101 (2008).

[21] Hulme, J. C., Doylend, J. K., Heck, M. J. R., Peters, J. D., Davenport, M. L., Bovington, J. T., Coldren, L. A., and Bowers, J. E., "Fully integrated hybrid silicon two dimensional beam scanner," Optics Express 23(5), 5861 (2015).

[22] Sun, J., Timurdogan, E., Yaacobi, A., Hosseini, E. S., and Watts, M. R., "Large-scale nanophotonic phased array," Nature 493(7431), 195-199 (2013). 
[23] Guo, W., Binetti, P. R., Althouse, C., Mašanović, M. L., Ambrosius, H. P., Johansson, L. A., and Coldren, L. A., "Two-dimensional optical beam steering with InP-based photonic integrated circuits," IEEE Journal on Selected Topics in Quantum Electronics 19(4) (2013).

[24] Acoleyen, K. V., Bogaerts, W., and Baets, R., "Two-Dimensional Dispersive Off-Chip Beam Scanner," IEEE Photon Technol. Lett. 23(17), 1270-1272 (2011).

[25] Verbist, M., Bogaerts, W., and Van Thourhout, D., "Design of weak 1-D bragg grating filters in SOI waveguides using volume holography techniques," Journal of Lightwave Technology 32, 1915-1920 (5 2014).

[26] Zhu, T., Hu, Y., Gatkine, P., Veilleux, S., Bland-Hawthorn, J., and Dagenais, M., "Arbitrary on-chip optical filter using complex waveguide Bragg gratings," Applied Physics Letters 108(10) (2016).

[27] Dumon, P., Priem, G., Nunes, L. R., Bogaerts, W., Van Thourhout, D., Bienstman, P., Liang, T. K., Tsuchiya, M., Jaenen, P., Beckx, S., Wouters, J., and Baets, R., "Linear and nonlinear nanophotonic devices based on silicon-on-insulator wire waveguides," Japanese Journal of Applied Physics, Part 1: Regular Papers and Short Notes and Review Papers 45, 6589-6602 (8 2006).

[28] Zhang, B., Dostart, N., Khilo, A., Brand, M., Qubaisi, K. A., Onural, D., Feldkhun, D., Popović, M. A., and Wagner, K., "Serpentine Optical Phased Array Silicon Photonic Aperture Tile with Two-Dimensional Wavelength Beam Steering," 2019 Optical Fiber Communications Conference and Exhibition, OFC 2019 Proceedings, M4E.5 (2019).

[29] Zhang, B., Dostart, N., Brand, M., Khilo, A., Feldkhun, D., and Popović, M. A., "Tiled Silicon-Photonic Phased Arrays for Large-Area Apertures," Conference on Lasers and Electro-Optics 2019 (CLEO), SF3N.2 (2019). 